\title{
Effect of Ceramic Brush Treatment on the Surface Quality and Edge Condition of Aluminium Alloy after Abrasive Waterjet Machining
}

\author{
Jakub Matuszak ${ }^{1 *}$ \\ 1 Faculty of Mechanical Engineering, Lublin University of Technology, ul. Nadbystrzycka 36, 20-618 Lublin, \\ Poland \\ * Corresponding author's email: j.matuszak@pollub.pl
}

\begin{abstract}
Material separation is one of the basic technological operations. Abrasive water jet machining (AWJM) is a universal cutting method; it is used for almost all structural materials, ranging from easy-to-cut materials such as aluminium alloys to materials that are difficult-to-cut after thermal treatment. In terms of assembly accuracy and product safety, surface quality and edge condition after cutting are of vital importance. Machining with ceramic brushes mounted on the machining centre is one of the methods for improving surface quality and removing burrs after AWJM. The aim of this study is to analyse the influence of machining conditions with ceramic brushes on the surface quality and effectiveness of deburring after abrasive water jet machining of EN AW-7075 aluminium alloy. For all tested brushing treatment conditions lower roughness parameters were obtained compared to the base surface after AWJM. A positive deburring effect was achieved for all fibre types after one brush pass. For the brush with the lowest stiffness fibres a rounded edge was obtained, while for the fibres with higher stiffness - the edges were chamfered.
\end{abstract}

Keywords: surface roughness, AWJM, brushing, deburring.

\section{INTRODUCTION}

Abrasive water-jet (AWJ) treatment is a very popular method of material separation, one that offers an alternative to typical cutting methods such as machining, gas cutting or laser beam cutting. The choice of cutting technology should take into account factors such as the type of workpiece material, thickness, machining performance, and cut surface quality. Due to its flexibility and a number of advantages, AWJ machining is widely used for cutting easy-to-cut materials (aluminium and magnesium alloys) and difficult-to-cut materials (stainless steels, titanium alloys) alike. AWJ cutting is based on the use of concentrated water jet energy. This energy is concentrated in a small area, which allows the separation of atomic bonds and the activation of erosion. The process is intensified by the addition of abrasive grains. One of the main advantages of water jet cutting is the absence of a heat-affected zone. Due to the minimal thermal impact, the process is dedicated to materials for which high processing temperature is unfavourable or not recommended, e.g. magnesium alloys that, due to their properties, may be subject to ignition in the classical processes of milling, cutting, turning, etc. [1]. The water abrasive jet cutting process is illustrated schematically in Figure 1.

Water 4 is supplied by a high-pressure pump. The medium in the mixing chamber 2 combines with the abrasive 3 and exits through the head nozzle in the form of an abrasive-water jet 1 . The erosion process is initiated when the jet hits the workpiece surface. Due to the deceleration of the jet at the workpiece exit side, the surface quality is lower on the exit side. Many studies focus on optimizing the AWJ process for improved surface quality after cutting. Mathematical modelling (Response Surface Methodology) can be used to optimize the process conditions such as surface roughness, material removal rate and hardness. In the study [2] three levels of variation were used for each of the studied parameters (abrasive feed, 




Fig. 1. Schematic view of the abrasive water jet process: 1 - abrasive water jet, 2 - mixing chamber, 3 - abrasive, 4 - high pressure water

stand-off distance and nozzle speed). The use of the Taguchi method to optimize machining of AZ91 magnesium alloy in order to achieve the lowest surface roughness was investigated in [3]. Surface roughness was analysed in three regions on the cut surface (initial damage, smooth and rough cutting zones). As shown by the (ANOVA) analysis, traverse speed had the greatest impact on surface roughness, while water pressure had the greatest impact on the depth of penetration. The authors of [4] used fuzzy logic to model the surface roughness of AZ91 alloy after AWJ cutting. Water pressure (WP), traverse speed (TS), and stand-off distance (SOD) were selected as parameters for optimization. The best results (37\% decrease in roughness) were observed for maximum pressure and minimum stand-off distance. Surface quality after cutting was analysed in [5]. To improve the surface quality after plasma cutting, the cut surfaces were subjected to centrifugal shot peening. Besides improved surface quality, another advantage of the process is that shot peening increases the microhardness of the surface layer. The Taguchi optimization method presented in [6] was employed to get the best appropriate combination of nine independent process parameters (feed rate, stand-off distance and abrasive flow rate) to find the minimum surface roughness in the cutting of metal matrix composite based on Al-6061 aluminium alloy. Optimal process parameters were achieved for the lowest stand-off distance $(0.5 \mathrm{~mm})$.

Due to the nature of the process, the condition of the edge on the entry side of the jet is adequate. The jet impact on the cut surface results in rounding of the edge. The value of the radius depends on the processing parameters and the properties of the workpiece material. However, when the water jet leaves the material, the edges remain sharp or the undesired phenomenon of burr formation may occur. Such condition of the edge requires performing an additional operation to remove the burr or to round or chamfer the edges.

There exist many methods of deburring, the most widely used being manual deburring, grinding and milling. One of the common methods is deburring of workpieces placed in abrasive media, including rotary-abrasive, vibratory finishing, abrasive flow machining, and abrasive blast-

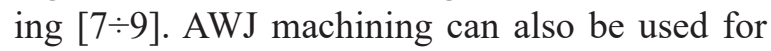
deburring, especially in hard-to-reach areas of workpieces $[10,11]$. Due to the degree of mechanization, the deburring process can be divided into manual, semi-automatic and automatic.

One of the methods dedicated to edge finishing is brushing. The brushing process is used when edge treatment and high surface quality are required. Popular applications for brushing include: deburring, as well as edge rounding, glossing, surface defect removal, surface cleaning and preparation $[12 \div 14]$. The brushing process can be used to remove surface defects such as scratches and grooves. The study [15] focused on the problem of detecting surface defects. The authors presented the method for defect detection using recurrence plots and recurrence quantifications. If defects cover at least 2.5 percent of the layer cross-section, they can be detected by cutting force data analysis. 
The authors of $[16,17]$ analysed forces during surface treatment with ceramic brushes. Ceramic brushes are characterized by low force value, which is desired when machining thin-walled components with reduced rigidity. Problems connected with machining thin-walled components are described in $[18,19]$.

Despite many research works devoted to the optimization of the AWJ machining process, in some situations the surface quality requires performing an additional operation to improve surface roughness and remove burrs, especially at the exit side of the jet. To that end, this study attempts to determine the effect of brushing treatment on the surface quality and edge condition after AWJ cutting.

\section{TEST METHODOLOGY}

The purpose of the study was to evaluate the effectiveness of surface quality improvement and deburring by brushing with ceramic fibre tools after AWJ machining. The overall experimental methodology is schematically shown in Figure 2.

A sheet of EN AW-7075 aluminium alloy plate with a thickness of $10 \mathrm{~mm}$ was used for testing. Table 1 shows the chemical composition and physical properties of the tested alloy.

First, the AWJ cutting process was conducted using constant parameters: feed rate $300 \mathrm{~mm} / \mathrm{min}$, stand of distance $3 \mathrm{~mm}$, abrasive flow rate $315 \mathrm{~g} /$ min. Samples with dimensions of $10 \times 15 \times 80$ were prepared. The experiment was divided into two stages. In the first stage, the effect of ceramic fibre stiffness on deburring and edge rounding efficiency was tested. The specimens were positioned in such a way that the adjacent surfaces forming the edge of the specimens were positioned at an angle of 45 degrees in relation to the axis of the tool, as shown in Figure 3a. The deburring process was carried out both from the entrance and exit sides of the abrasive water jet. Edge conditions after AWJ cutting and

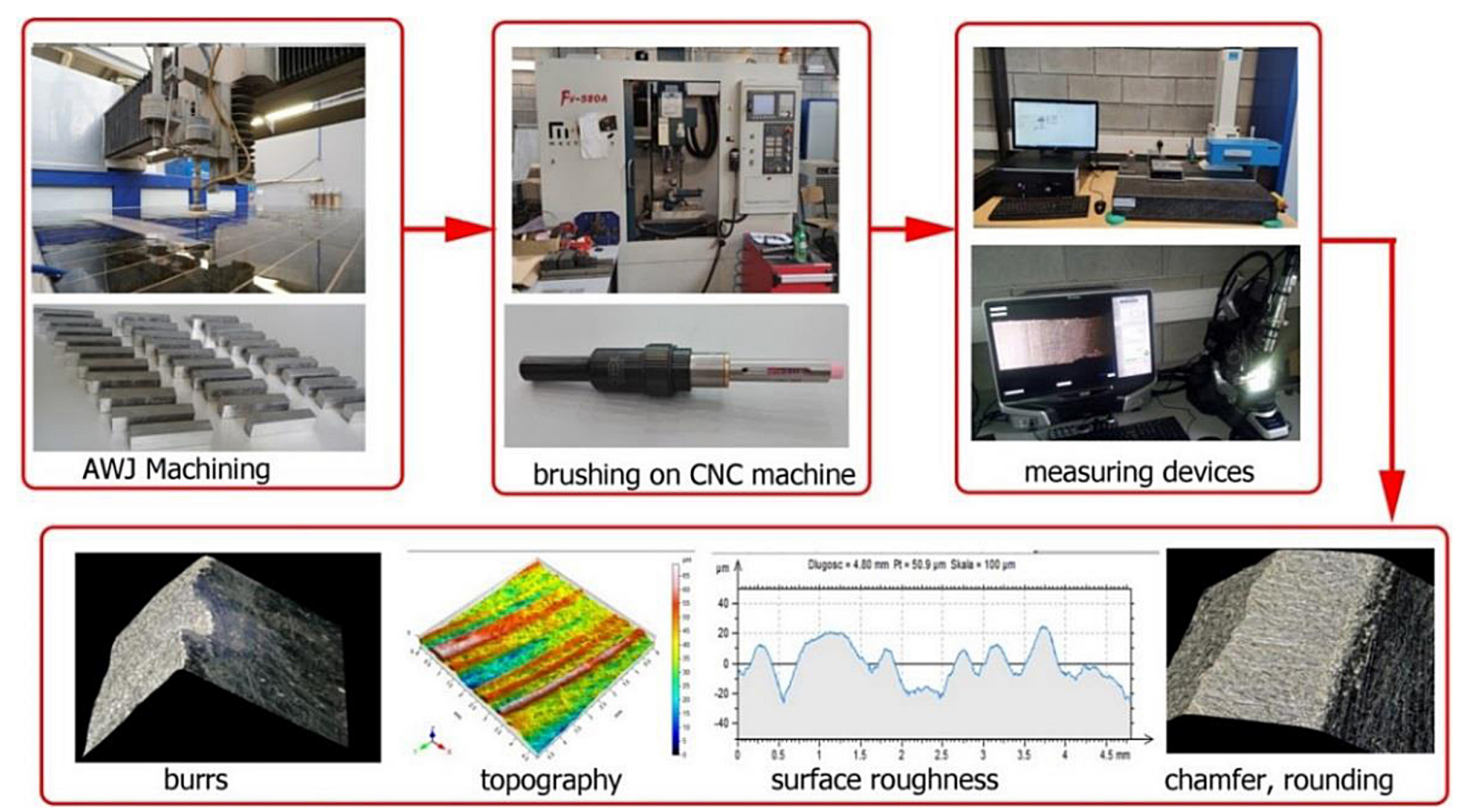

Fig. 2. Stage diagram of the research experiment

Table 1. Chemical composition and physical properties of EN AW-7075

\begin{tabular}{|c|c|c|c|c|c|c|c|c|}
\hline \multicolumn{9}{|c|}{ Chemical composition, Wt.\% } \\
\hline $\mathrm{Cu}$ & $\mathrm{Mn}$ & $\mathrm{Mg}$ & $\mathrm{Cr}$ & $\mathrm{Zn}$ & $\mathrm{Si}$ & $\mathrm{Fe}$ & $\mathrm{Ti}$ & $\mathrm{Al}$ \\
\hline 1.59 & 0.01 & 2.56 & 0.18 & 5.78 & 0.07 & 0.13 & 0.05 & Rest \\
\hline \multicolumn{9}{|c|}{ Physical properties } \\
\hline \multicolumn{3}{|c|}{$\begin{array}{c}\mathrm{Rm} \\
\mathrm{MPa}\end{array}$} & \multicolumn{3}{|c|}{$\begin{array}{l}\mathrm{Rp}_{0.2} \\
\mathrm{MPa}\end{array}$} & \multicolumn{3}{|c|}{$\mathrm{HB}$} \\
\hline \multicolumn{3}{|c|}{599} & \multicolumn{3}{|c|}{488} & \multicolumn{3}{|c|}{172} \\
\hline
\end{tabular}



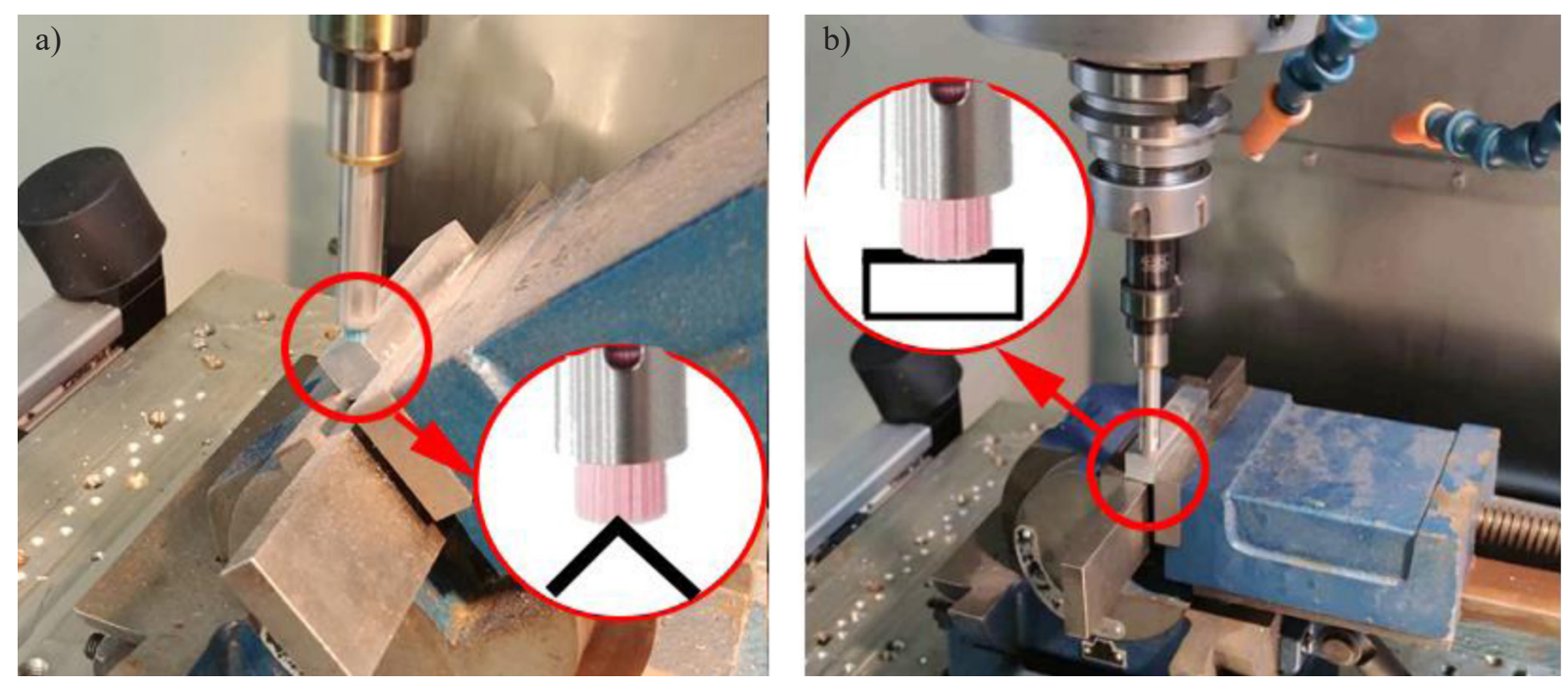

Fig. 3. Workpiece orientation in relation to the tool: a) during surface roughness experiment, b) during deburring experiment

brushing were measured using a Keyence VHX 5000 digital microscope. The second part of the experiment was to analyse the effect of the brushing treatment on the surface roughness that was formed after AWJ cutting. The surfaces after AWJ cutting were set perpendicular to the brush axis (Fig. 3b).

To intensify the process, 1,5 and 10 brush passes were used on the surface. Details of the experimental investigation of the effect of brushing on deburring efficiency and surface roughness are given in Table 2. The adopted brushing parameters are in accordance with the manufacturer's recommendations.
Ceramic fibres are characterized by different stiffness. The pink A13 fibres have the highest flexibility, while the blue A31 fibres are the most rigid.

Roughness was measured in the smooth and rough zones with the use of the Taylor-Hobson Surtronic $3+$ device and T8000RC120-400 profilographometer. Analysis of variance (ANOVA) was used to test the effect of brushing conditions on surface roughness with a significance level of 0.05 . The experiment was repeated six times. For each edge, five measurements were made of the edge radius or chamfer.

Table 2. Brushing conditions

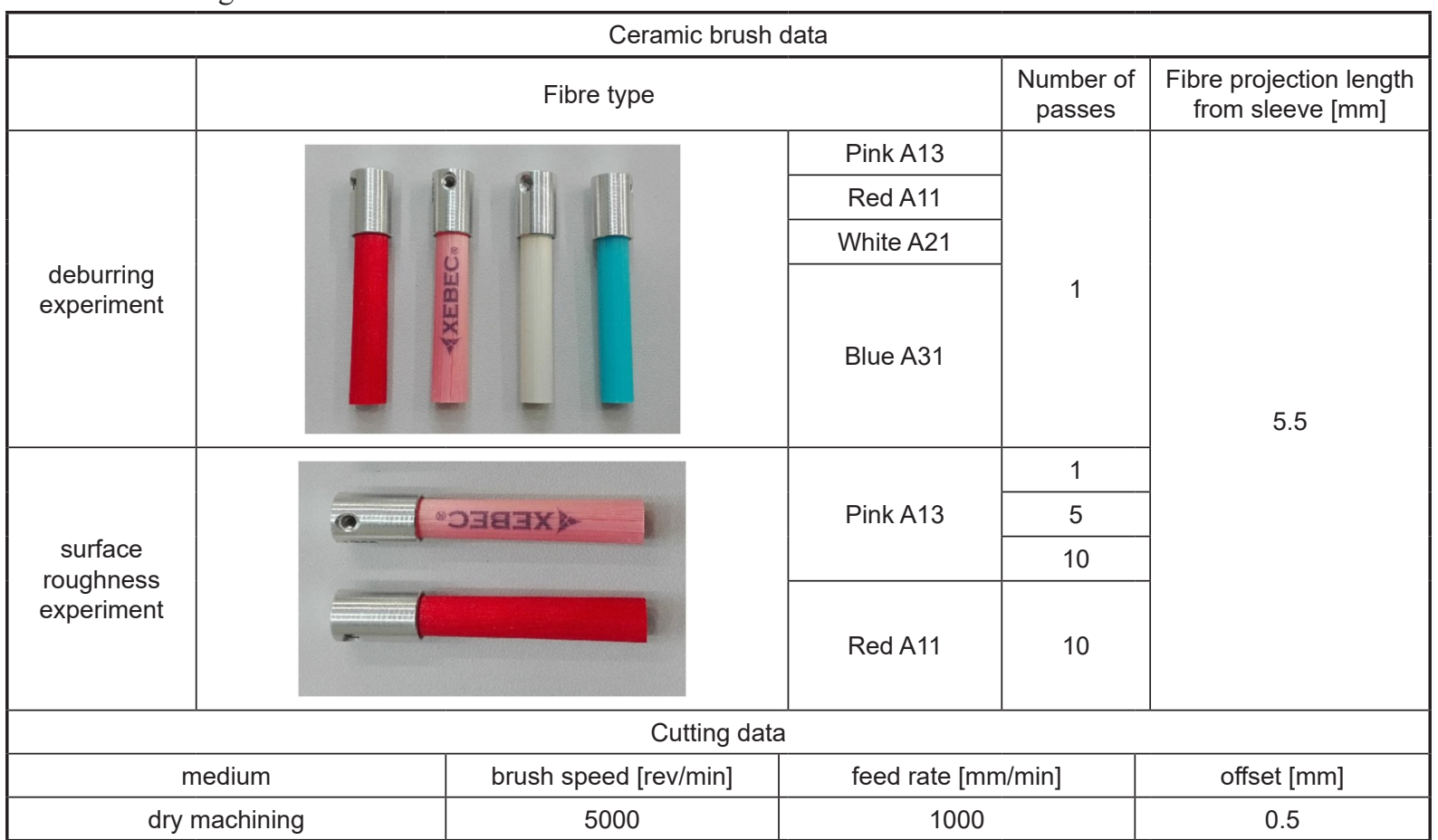




\section{RESULTS}

Due to the nature of the AWJ cutting process, burrs are formed on the edges from the side of the smooth zone, as shown in Figure 4, where the water-abrasive jet exits the workpiece. Consequently, an additional process must be performed to mitigate this undesired phenomenon.

Due to the efficiency and effectiveness of deburring and rounding the edges with ceramic brushes, one pass along the brushing edge was used.

\section{Impact of brushing on edge conditions}

Table 3 shows the views of the edge after brushing, both on the smooth and on the rough side. Edge chamfering was observed with the A11, A21 and A31 filament brush treatment, while edge rounding was obtained after the A13 filament brush treatment.

The stiffer fibres A11, A21 and A31 caused shearing of the material layer and a chamfer, while the flexible fibres were bent when impacted on the workpiece surface, which resulted in a rounded edge.
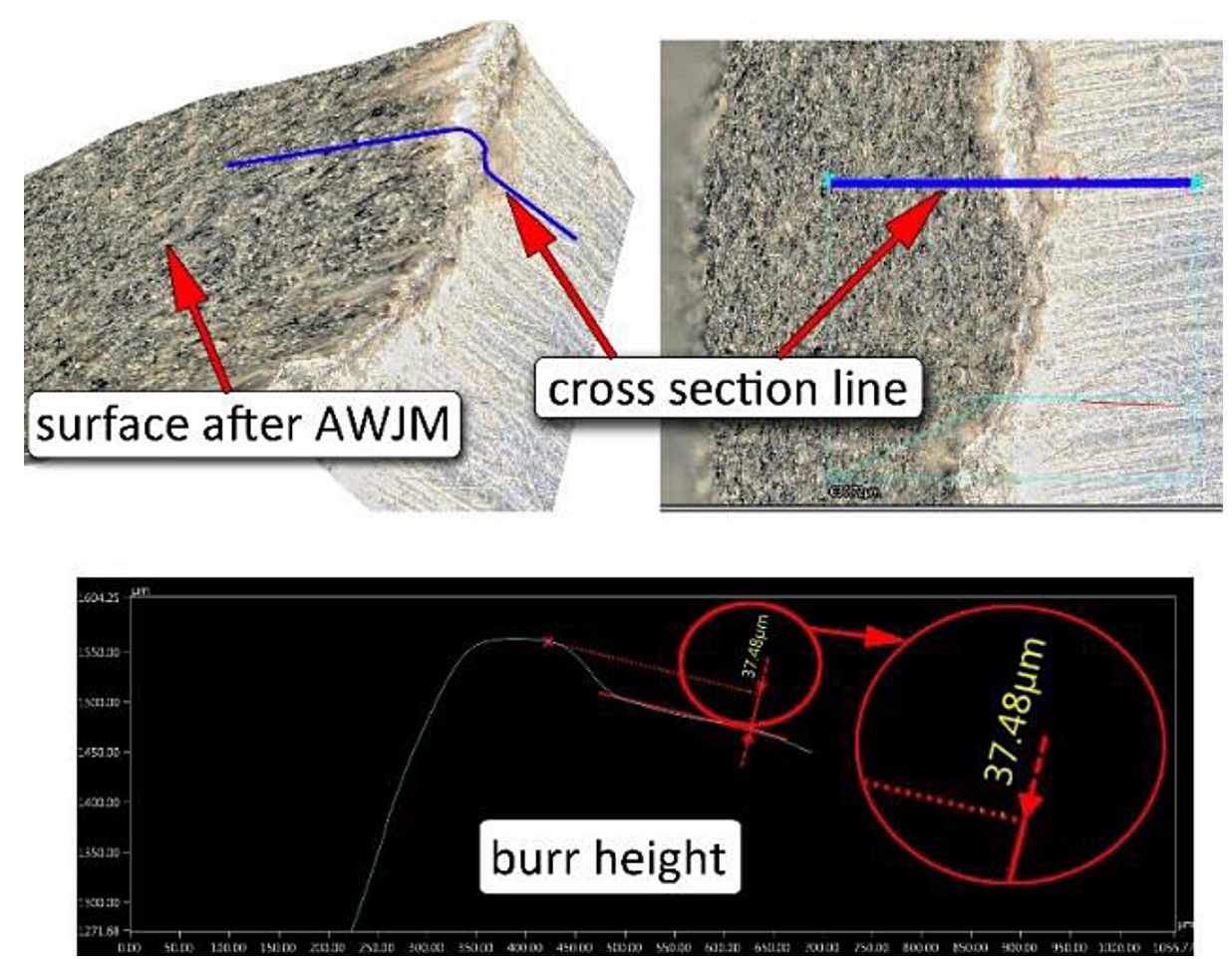

Fig. 4. Burr height in the rough zone

Table 3. View of the edges after the deburring process

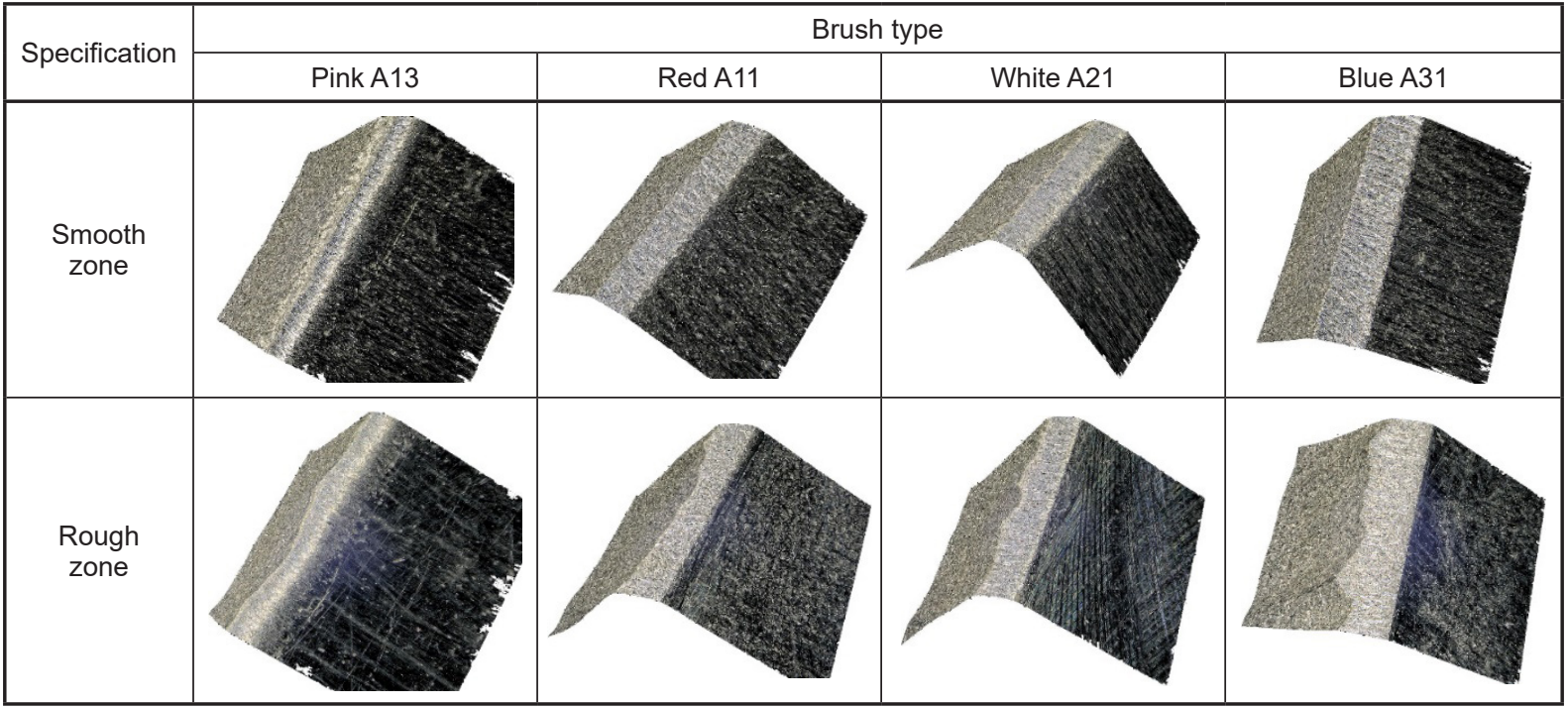


Figure 5 shows the value of edge radius after brushing in the rough and smooth zones. It can be observed that the energy of the abrasive water jet in the entry zone caused rounding of the edges, the mean value of the rounding being $88 \mu \mathrm{m}$.

The use of the brushing treatment in the entrance area led to an increase in the radius of the rounded edges. However, burrs were formed on the edges in the rough zone (Fig. 4). Some of the brush filament energy was converted into the deburring process, which explains the smaller edge radius in the exit zone.

Figure 6 shows the value of the chamfer in the smooth and rough zones after the treatment with brushes A11, A21, A31. It can be observed that as the stiffness of the fibres increases, the intensity of the brush impact on the treated edge increases too, leading to an increased edge chamfer. Smaller chamfer values can be observed in the exit zone. Some of the

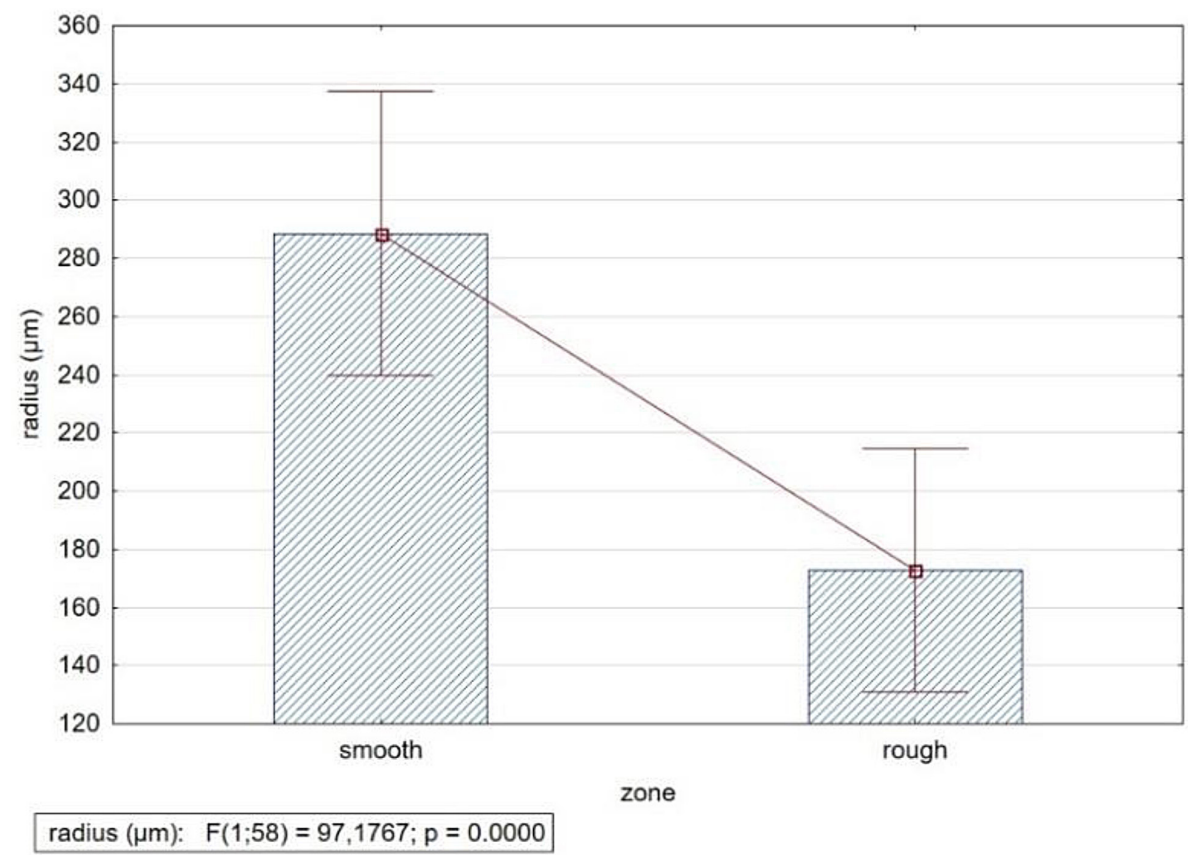

Fig. 5. Edge radius after brushing in the rough and smooth zones

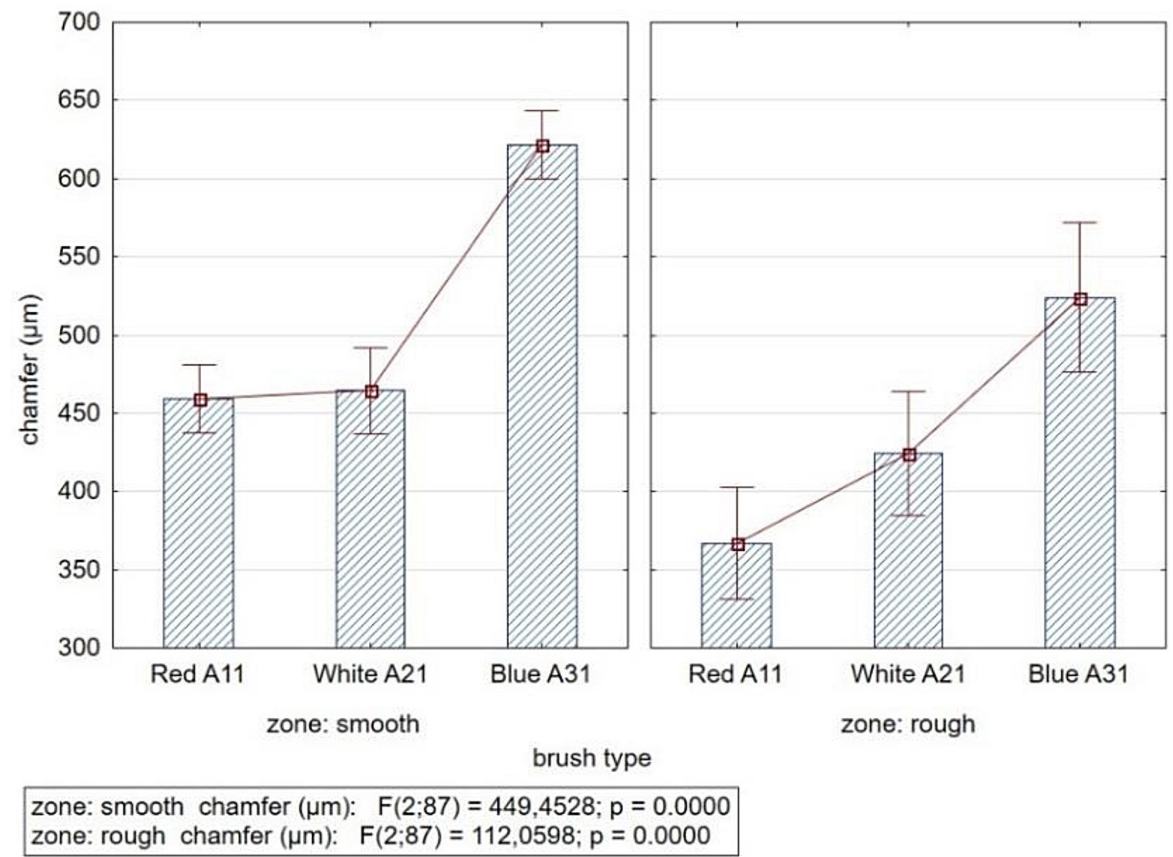

Fig. 6. Edge chamfer after brushing treatment with different types of fibres 
energy of the fibres was used for deburring in the rough zone.

Higher values of the standard deviation result from uneven edges (characteristic striations after AWJ treatment) and the formation of burrs of different heights. The ANOVA results confirm the statistically significant influence of the number of brush passes on the chamfer value. F-ratios $(F=449$ in smooth zone and $\mathrm{F}=112$ in rough zone) are greater than $F$ - critical $\left(F=3.10\right.$ for $\mathrm{df}_{1}=2$ and $\left.\mathrm{df}_{2}=87\right)$.

\section{Impact of brushing on surface roughness}

Surface topography after cutting with water abrasive jet is shown in Figure 7. Characteristic stripes are visible, especially in the rough zone.
Table 4 shows the views of surface topography after brushing with the A13 pink filament brush. There is a visible decrease in the value of the Sa roughness parameter with an increase in the number of tool passes.

The use of higher stiffness fibres (A11 Red) leads to a significant decrease in surface roughness. In addition, there are characteristic curved marks reflecting the movement of the fibres, which indicates the removal of topography following the AWJ treatment (Fig. 8).

Table 5 shows the surface roughness after brushing with the A13 pink filament brush in relation to the roughness after AWJ cutting.

Figure 9 shows the effect of the number of passes on the surface roughness in the smooth and rough a)

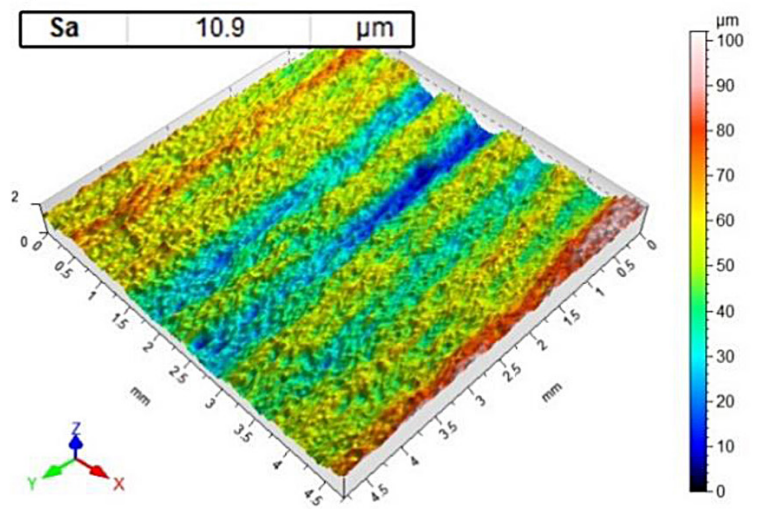

b)

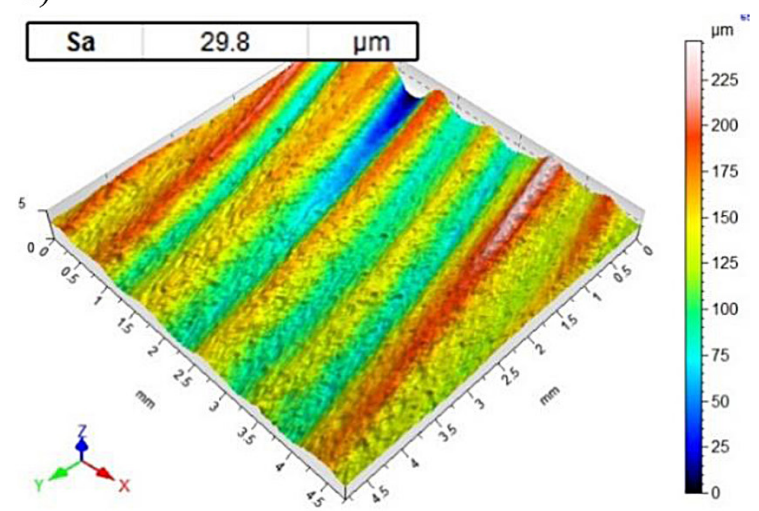

Fig. 7. Surface topography after AWJ cutting: a) smooth zone, b) rough zone

Table 4. Surface topography after brushing with Pink A13 filament

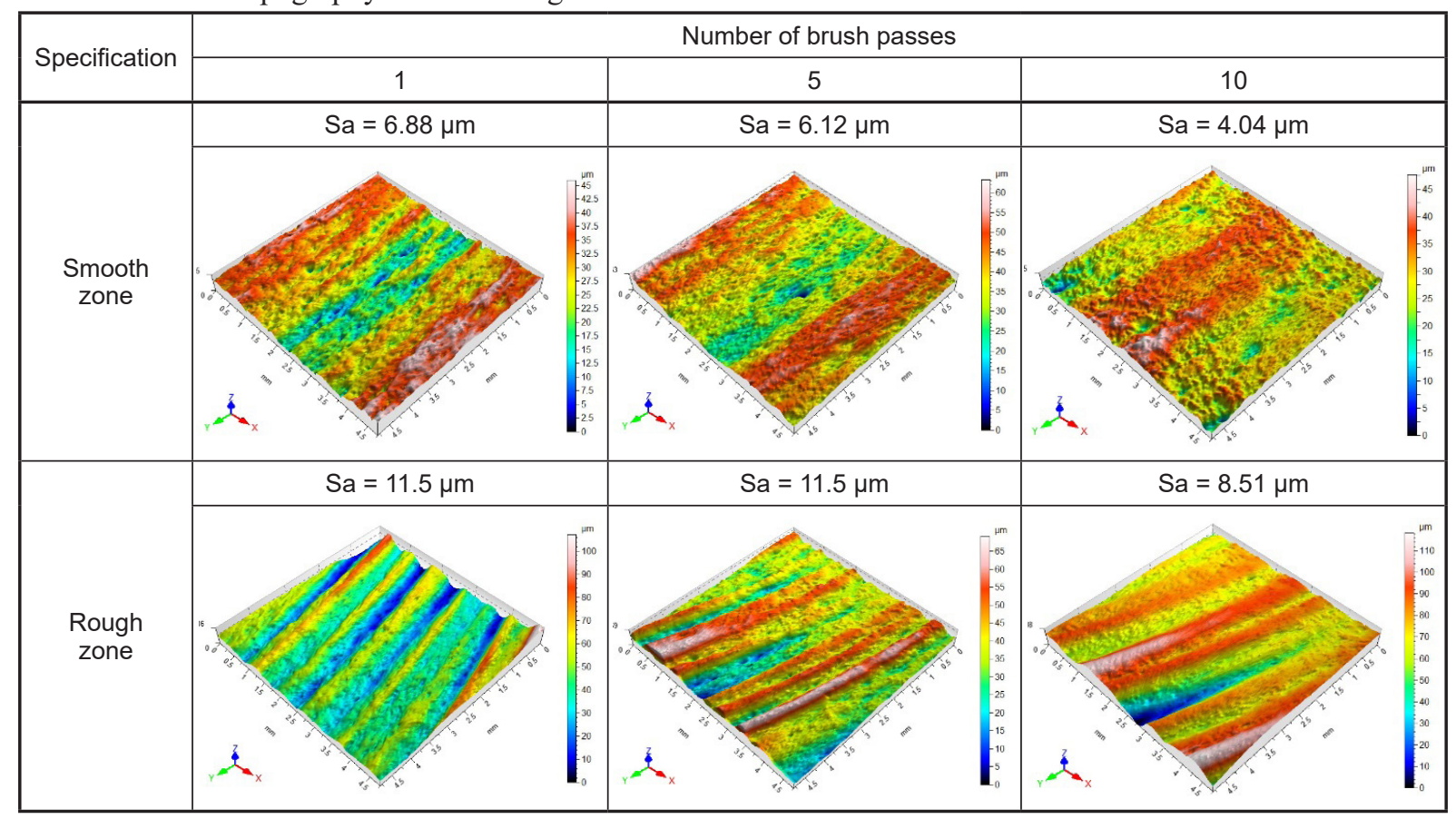


a)

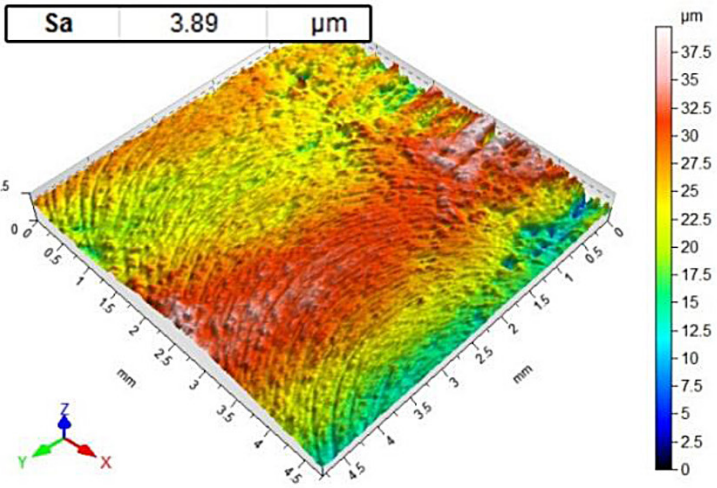

b)

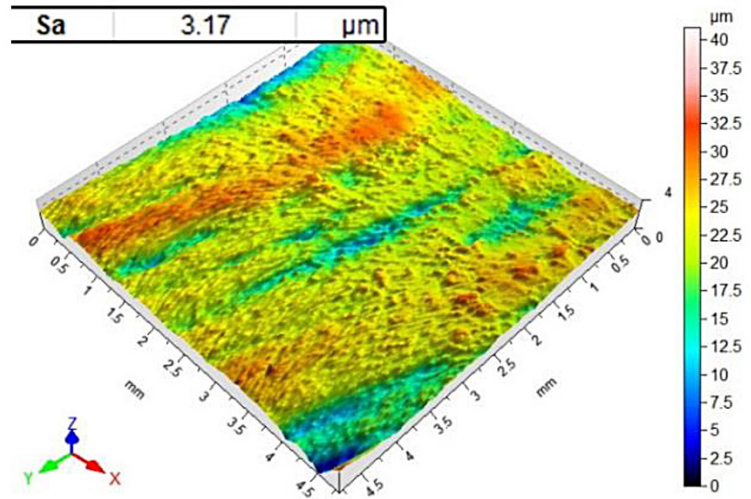

Fig. 8. Surface topography after brushing with Red A11: a) smooth zone, b) rough zone

Table 5. Surface roughness after brushing with Pink A13 filament

\begin{tabular}{|c|c|c|c|c|c|}
\hline \multicolumn{7}{|c|}{ Smooth zone } & \multicolumn{3}{c|}{ Rough zone } \\
\hline \multicolumn{7}{|c|}{ Ra $=5.9 \mu \mathrm{m}$} & $\mathrm{Ra}=15.5 \mu \mathrm{m}$ \\
\hline \multicolumn{7}{|c|}{ After AWJ cutting } \\
\hline 1 pass & 5 passes & 10 passes & 1 pass & 5 passes & 10 passes \\
\hline $\mathrm{Ra}=4.99 \mu \mathrm{m}$ & $\mathrm{Ra}=4.33 \mu \mathrm{m}$ & $\mathrm{Ra}=4.20 \mu \mathrm{m}$ & $\mathrm{Ra}=15.17 \mu \mathrm{m}$ & $\mathrm{Ra}=13.17 \mu \mathrm{m}$ & $\mathrm{Ra}=11.08 \mu \mathrm{m}$ \\
\hline
\end{tabular}

zones. The average roughness value in the entrance zone after AWJ cutting is $\mathrm{Ra}=5.9 \mu \mathrm{m}$, while in the exit zone it is $\mathrm{Ra}=15.5 \mu \mathrm{m}$. There is a slight decrease in roughness after one brush pass. After 10 brush passes, the roughness is reduced by about $30 \%$ compared to the roughness after AWJ cutting.

Table 6 shows the surface roughness after brushing with the A13 pink and A11 red filaments.
Figure 10 shows the effect of the type of brush fibre on the surface roughness of specimens. The use of stiffer Red A11 fibre allows for a significant reduction in surface roughness. Values of the F statistic prove a significant influence of the fibre type on the surface roughness of specimens. F-ratios $(\mathrm{F}=130$ in smooth zone and $\mathrm{F}=38.5$ in rough zone) are greater than $\mathrm{F}$ - $\operatorname{critical}\left(\mathrm{F}=4.20\right.$ for $\mathrm{df}_{1}=1$ and $\left.\mathrm{df}_{2}=28\right)$.
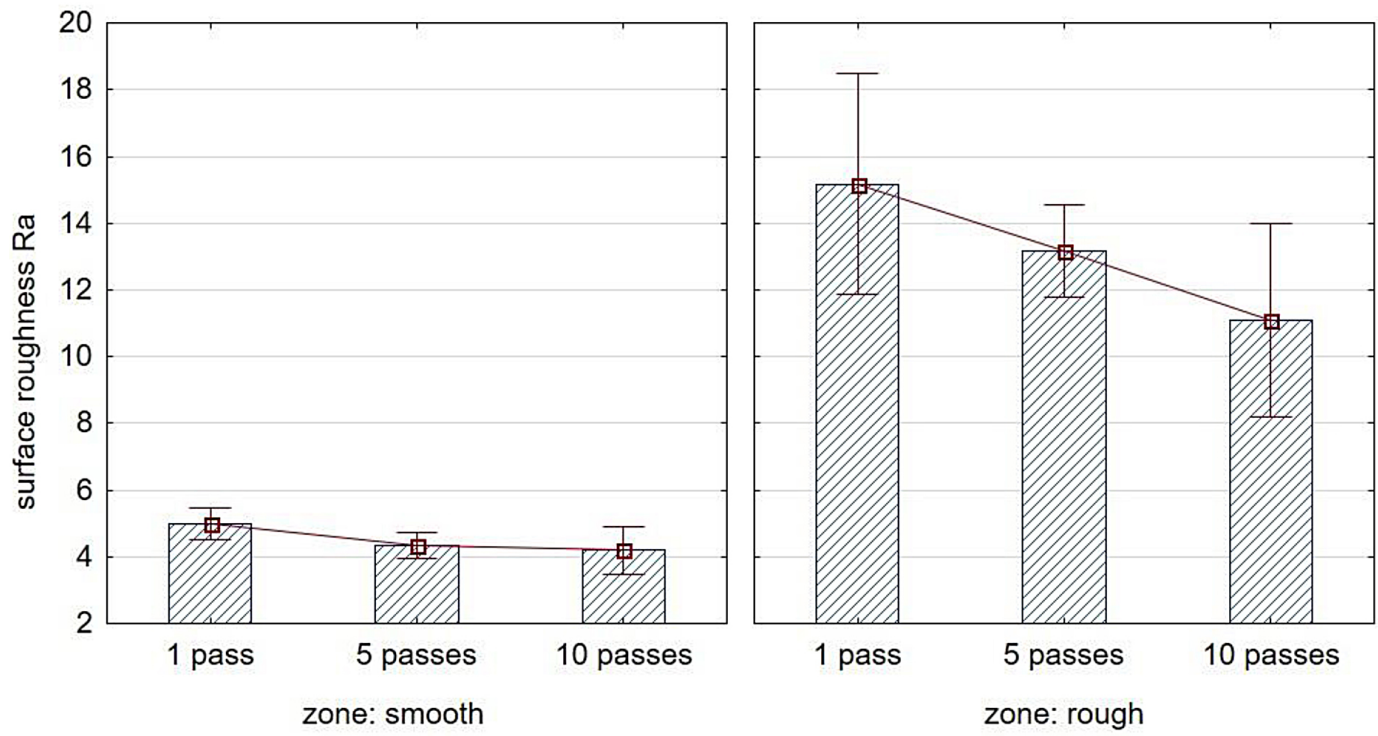

zone: smooth surface roughness Ra: $F(2 ; 42)=9,0627 ; p=0,0005$
zone: rough surface roughness Ra: $F(2 ; 42)=8,872 ; p=0,0006$

Fig. 9. Effect of the number of brush passes on the surface roughness of specimens (A13 pink brush filament) 
Table 6. Surface roughness after brushing with Pink A13 and Red A11 filaments

\begin{tabular}{|c|c|c|c|}
\hline \multicolumn{2}{|c|}{ Smooth zone } & \multicolumn{2}{c|}{ Rough zone } \\
\hline Pink A13 & Red A11 & Pink A13 & Red A11 \\
\hline $\mathrm{Ra}=4.20 \mu \mathrm{m}$ & $\mathrm{Ra}=1.85 \mu \mathrm{m}$ & $\mathrm{Ra}=11.08 \mu \mathrm{m}$ & $\mathrm{Ra}=5.93 \mu \mathrm{m}$ \\
\hline
\end{tabular}
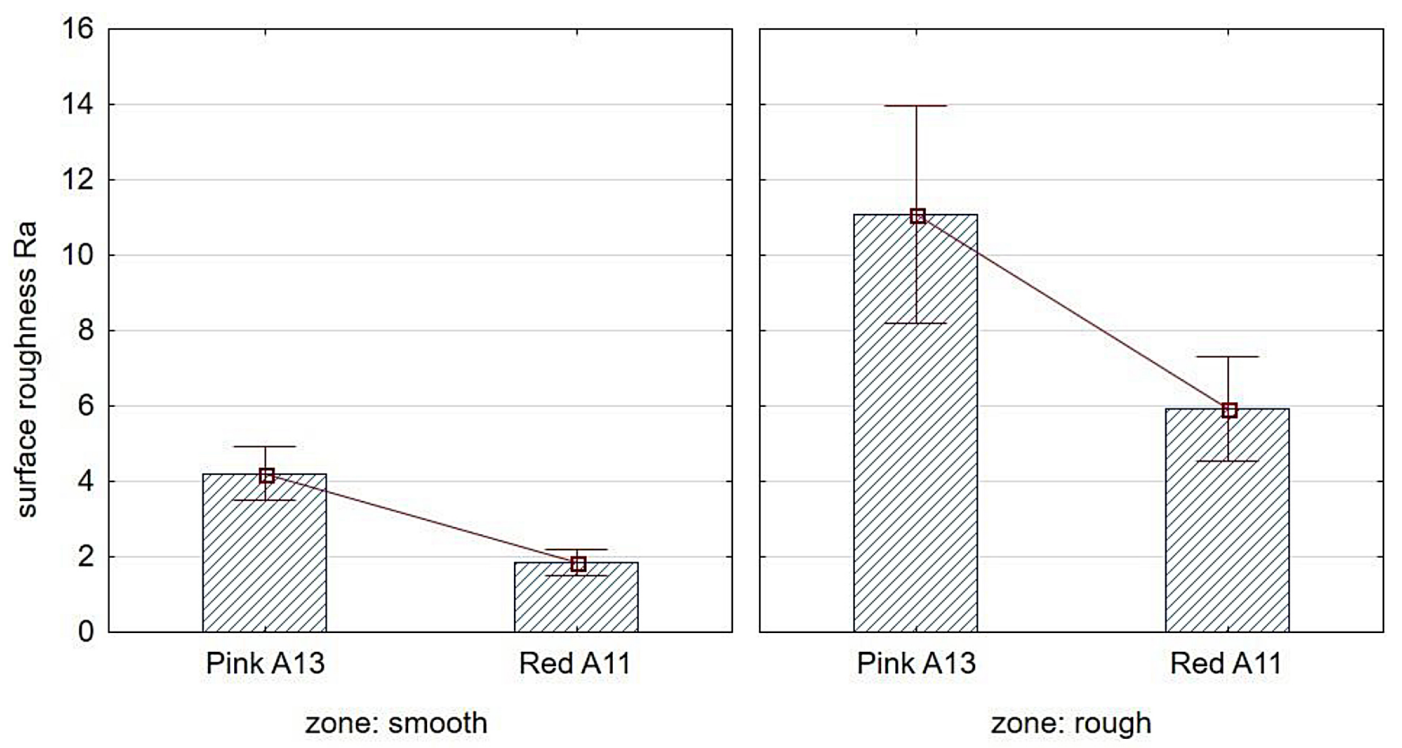

zone: smooth surface roughness $\mathrm{Ra}: \mathrm{F}(1 ; 28)=129,9521 ; \mathrm{p}=0.0000$ zone: rough surface roughness Ra: $F(1 ; 28)=38,5048 ; p=0,00000$

Fig. 10. Effect of brush filament on the surface roughness of specimens

\section{CONCLUSIONS}

The experimental tests investigating the impact of machining with ceramic filaments brushes of EN AW- 7075 aluminium alloy samples after AWJ cutting on deburring effectiveness and surface roughness quality have led to the following conclusions:

- burrs appear on the edges of the specimens after water jet cutting in the rough zone;

- the surface after water jet cutting has a very non-uniform roughness;

- the stiffness of the fibres influences the edge condition after processing: for low stiffness fibres (pink A13) a rounding was obtained, while for stiffer fibres the edges were chamfered;

- edge burrs can be removed with a single pass of the tool brush;

- brushing reduces the surface roughness after water abrasive jet cutting;

- ANOVA analysis confirmed a significant effect of brush type and the number of brush passes on the surface roughness and the rounding and chamfering value.

For efficient machining, it is desirable that the deburring process be carried out as quickly as possible. In all cases, the burrs were removed after one pass of the brushing tool. However, improving surface roughness sometimes requires more passes.

\section{REFERENCES}

1. Zagórski I., Kłonica M., Kulisz M., Łoza K. Effect of the AWJM Method on the Machined Surface Layer of AZ91D Magnesium Alloy and Simulation of Roughness Parameters Using Neural Networks. Materials. 2018;11:2111. DOI: 10.3390/ ma11112111.

2. Joel C., Jeyapoovan T., Praneeth Kumar P. Experimentation and Optimization of Cutting Parameters of Abrasive Jet Cutting on AA6082 through Response Surface Methodology. Mater. Today Proc. 2021;44:3564-3570. DOI: 10.1016/j.matpr.2020.09.452.

3. Niranjan C.A., Srinivas S., Ramachandra M. Effect of Process Parameters on Depth of Penetration and Topography of AZ91 Magnesium Alloy in Abrasive Water Jet Cutting. J. Magnes. Alloys. 2018;6:366-374. DOI: 10.1016/j.jma.2018.07.001.

4. Srinivasan R., Jacob V., Muniappan A., Madhu S., Sreenevasulu M. Modeling of Surface Roughness in Abrasive Water Jet Machining of AZ91 Mag- 
nesium Alloy Using Fuzzy Logic and Regression Analysis. Mater. Today Proc. 2020;22:1059-1064. DOI: 10.1016/j.matpr.2019.11.295.

5. Zaleski K., Skoczylas A., Ciecieląg K. The Investigations of the Surface Layer Properties of C45 Steel After Plasma Cutting and Centrifugal Shot Peening. In; 2020;172-185.

6. Maneiah D., Shunmugasundaram M., Raji Reddy A. Zareena Begum Optimization of Machining Parameters for Surface Roughness during Abrasive Water Jet Machining of Aluminium/Magnesium Hybrid Metal Matrix Composites. Mater. Today Proc. 2020;27:1293-1298. DOI: 10.1016/j.matpr.2020.02.264.

7. Bergs T., Müller U., Barth S., Ohlert M. Experimental Analysis on Vibratory Finishing of Cemented Carbides. Manuf. Lett. 2021;28:21-24. DOI: 10.1016/j.mfglet.2021.02.004

8. Aurich J.C., Dornfeld D., Arrazola P.J., Franke V., Leitz L., Min S. Burrs-Analysis, Control and Removal. CIRP Ann. 2009;58:519-542. DOI:10.1016/j.cirp.2009.09.004.

9. Swat M., Brünnet H., Lyubenova N., Schmitt J., Diebels S., Bähre D. Improved Process Control and Model of Axial Forces of One-Way Abrasive Flow Machining. Procedia CIRP. 2014;14:19-24, DOI: 10.1016/j.procir.2014.03.106.

10. Kwon B.C., Kim K.H., Kim K.H., Ko S.L. New Abrasive Deburring Method Using Suction for Micro Burrs at Intersecting Holes. CIRP Ann. - Manuf. Technol. 2016;65:145-148. DOI: 10.1016/j. cirp.2016.04.085.

11. Yang M., Choi J., Lee J., Hur N., Kim D. Wet Blasting as a Deburring Process for Aluminum. J. Mater. Process. Technol. 2014;214:524-530. DOI:10.1016/j.jmatprotec.2013.09.011.
12. Stango R.J. Filamentary Brushing Tools for Surface Finishing Applications. Met. Finish. 2002;100:8291. DOI: 10.1016/S0026-0576(02)82007-4.

13. Novotný F., Horák M., Starý M. Abrasive Cylindrical Brush Behaviour in Surface Processing. Int. J. Mach. Tools Manuf. 2017;118-119:61-72. DOI: 10.1016/j.ijmachtools.2017.03.006.

14. Matuszak J., Zaleski K. Analysis of Deburring Effectiveness and Surface Layer Properties around Edges of Workpieces Made of 7075 Aluminium Alloy. Aircr. Eng. Aerosp. Technol. 2018;90:515523. DOI:10.1108/AEAT-05-2016-0074.

15. Ciecieląg K., Kecik K., Zaleski K. Defects Detection from Time Series of Cutting Force in Composite Milling Process by Recurrence Analysis. J. Reinf. Plast. Compos. 2020;39:890-901. DOI: $10.1177 / 0731684420935985$.

16. Matuszak J., Klonica M., Zagorski I. Effect of Brushing Conditions on Axial Forces in Ceramic Brush Surface Treatment. In Proceedings of the 2019 IEEE 5th International Workshop on Metrology for AeroSpace (MetroAeroSpace); IEEE: Torino, Italy, June 2019, 644-648.

17. Matuszak J., Kłonica M., Zagórski I. Measurements of Forces and Selected Surface Layer Properties of AW-7075 Aluminum Alloy Used in the Aviation Industry after Abrasive Machining. Materials 2019;12:3707. DOI: 10.3390/ma12223707.

18. Zawada-Michałowska M., JózwikJ., Legutko S., Mika D., Pieśko P., Pytka J. Cutting Force during Surface Layer Milling of Selected Aluminium Alloys. Materials. 2020;13:5725. DOI: 10.3390/ma13245725.

19. Rusinek R., Zaleski K. Dynamics of Thin-Walled Element Milling Expressed by Recurrence Analysis. Meccanica 2016, 51, 1275-1286. DOI: 10.1007/ s11012-015-0293-y. 